\title{
Klassifikation von Schutthalden
}

\section{Zusammenfassung}

Die vorliegende Klassifikation von Schutthalden ist ein Versuch, ihren Formenreichtum zu überblicken. Immer wieder werden zum Teil sich widersprechende Angaben über das Aussehen, vor allem über die Neigungsverhältnisse und die Korngrößenanordnung von Schutthalden gemacht, die wohl richtig sind, aber sich nur auf einen bestimmten Typ anwenden lassen. Es gibt nur ganz wenig Merkmale, welche allgemein für Schutthalden gültig sind.

Die hier vorgelegte Klassifikation kann dazu dienen, Einzelbeobachtungen sinngemäß einer bestimmten Schutthaldenart zuzuordnen. Sie beschränkt sich auf Schutthalden des humiden Klimas, vor allem der Alpen.

\section{Abstract}

Classification of scree slopes. The present classification of scree slopes is an attempt at a review of the various possible forms. In the literature, many data on the form, slope angles and grain sizes of scree slopes are found which may be correct, but which are only applicable to certain types of scree slopes. There are only very few characteristic features which are generally applicable for scree slopes.

The classification of scree slopes presented here can be used to relate individual observations to specific types of scree slopes.

\section{Definition und Abgrenzung gegen ähnliche Erscheinungen}

Schutthalden entstehen durch vorwiegend trockene Schüttung von Lockermaterial. Unter Schüttung verstehen wir eine besondere Art von Massenbewegung, bei der Lockermaterial durch rasches Gleiten, Rollen oder Springen der Einzelstücke auf einer Halde zum Ablagern kommt. Eine Schutthalde setzt also ein steileres Obergehänge, einen Wandhang, voraus, auf dessen Oberfläche sich Lockermaterial nicht halten kann und auf welchem der nötige Impuls für eine rasche Bewegung erteilt wird.

Zum Aufbau einer typischen Schutthalde muß die Zufuhr aus dem Obergehänge in Form von einzelnen
Bruchstücken oder kleineren Mengen von Detritus erfolgen. Sobald größere Schuttmassen niederstürzen oder abgleiten, erfolgt die Bewegung im Verband. Dann entstehen nicht Schutthalden, sondern Felssturz- und Bergsturzablagerungen, die sich in ihren typischen Formen von Schutthalden wesentlich dadurch unterscheiden, daß sie kein einheitliches Gefälle haben und oft bis in einen Flachboden vorstoßen, wo sie sich ausbreiten.

Die Schüttung von kohäsionslosem Material (Einzelbruchstücke oder kleinere Quantitäten von grobem Detritus) führt zur Ausbildung einer Halde, welche durch einen von der Beschaffenheit des verfrachteten Materials abhängigen maximalen Böschungswinkel charakterisiert ist.

Stammt das Material aus der fortschreitenden Verwitterung einer geschlossenen Wand, so entsteht eine breit geschüttete Schutthalde, die Schutthalde im engeren Sinn. Wird das Material zuerst in einer Steinschlagrinne gesammelt und am Ausgang der Rinne geschüttet, so bildet sich ein Schuttkegel. Wir werden, um nicht immer beide Formen aufzählen zu müssen, den Begriff Schutthalde als Oberbegriff verwenden, der Schutthalden im engeren Sinn und Schuttkegel umfaßt.

Durch die Schüttung aus einem Obergehänge kann die Schutthalde von einer Blockhalde unterschieden werden, in welcher der Schutt an Ort und Stelle durch Verwitterung entstanden ist und aus Residualschutt besteht. Solche Blockhalden können Hänge bilden, die weit flacher sind als die Maximalböschung ihres Materials. Sie unterscheiden sich von Schutthalden um so weniger, je weniger ihre Böschung von der Maximalböschung abweicht, zeigen aber doch nicht die Materialsortierungen, die für Schutthalden charakteristisch sind.

Eine Sonderstellung nehmen jene steilen Halden ein, in die ein Gletscher Schutt schiebt. Meist handelt es sich um Hänge- oder Kargletscher. Von den bisher geschilderten Schüttungsarten unterscheidet sich diese dadurch, da $\beta$ der Schutt nicht direkt aus einer Felswand stammt, aber auch nicht aus einer Steinschlagrinne kommt, sondern als Fallschutt zunächst vom Gletscher transportiert und, mit Erosionsmaterial vom Gletscherbett vermischt, an der

Dr. E. Gerber, 5107 Schinznach-Dorf 
Stirne ausgestoßen wird. Die Schutthalde hat die Breite der Gletscherstirn und die Schuttzufuhr hört auf, sobald sich der Gletscher zurückzieht. Solche Gletscherschutthalden sind in den Alpen nicht selten.

Durch den Zusatz «vorwiegend» zu «trockener Schüttung» soll festgehalten werden, daß im humiden Klima am Aufbau einer Halde immer Wasser mitbeteiligt ist. Bei Schutthalden im engeren Sinn, die unter geschlossenen Felswänden aufgebaut werden, ist zwar trockene Schüttung weitgehend gegeben, aber beim Weitertransport in der Halde, beim Konsolidieren und Dichtschlämmen spielt Wasser doch eine wichtige Rolle, erst recht beim Verwittern und der Besiedlung durch höhere Pflanzen.

Unvermeidlich ist beim Aufbau von Schuttkegeln die Mitwirkung von Wasser und Schnee, so daß alle Übergänge von Schuttkegeln zu Schwemmkegeln beobachtet werden können und auch gemischte Formen entstehen. Beim Wassertransport gelangt das Material zur Ablagerung, wenn die Schleppkraft nachläßt, wobei Feinmaterial am weitesten transportiert wird. In den Schuttrinnen kommt es immer wieder zu Stauungen, so daß sich das Material in Schüben auf den Kegel ergießt und Schuttströme, oft von Murgangcharakter, auf der Halde zur Ablagerung kommen.

Eine weitere Sonderstellung nehmen Schuttkegel am $F u ß$ von Wasserfällen ein, die, wenn genügend Wasser abstürzt, ausgekolkt sind, so $\mathrm{da} B$ sich ein Hohlkegel ergibt, der dort, wo Wasser austritt, in einen Schwemmkegel übergeht.

Durch die ganz andere Entstehungsart, die sich in der Form und Neigung äußert, vor allem durch die moränenartige Kornverteilung, unterscheiden sich Blockströme von Schutthalden. Auch Stirn- und Seitenmoränen bestehen zum Teil aus Fallschutt, der aber nicht direkt geschüttet wurde, so daß das Charakteristikum echter Schutthalden, die Oberflächenschicht aus kohäsionslosem Schutt mit maximaler Böschung fehlt. Durch die Beimengung von Feinmaterial sind Moränen meist kohärent, so daß vor allem auf der Innenseite, wenn sich der Gletscher zurückzieht, Wände freigelegt werden, an deren Fuß sich dann durch Zufall Schutthalden bilden, wie dies ja auch bei Kieswänden der Fall sein kann. Schutthalden gehören zu der großen Gruppe der potentiellen Schuttmantelhänge. Diese werden definiert als Hänge, auf denen sich kohäsionsloser Schutt halten kann. Da es auch Hänge geringer Neigung gibt, die keinen Schuttmantel tragen, ist der Zusatz «potentiell» notwendig.

In der Gruppe der potentiellen Schuttmantelhänge gehören die Schutthalden zu den steilsten, da bei der Schüttung die Maximalböschung von kohäsionslosem Lockergestein entsteht.

Der maximale Böschungswinkel ist vom Material, vor allem von seiner Form und Rauhigkeit abhängig und bildet eines der Merkmale, das sich für eine Klassifikation eignet.

Den potentiellen Schuttmantelhängen stehen die Wandhänge gegenüber, die so steil sind, daß sich einmal in Bewegung gesetzter Lockerschutt nicht halten kann und rasch abgleitet oder abstürzt. Wandhänge setzen kohärentes Material voraus und bestehen in ihrer typischen und verbreitetsten Ausbildung aus Festgestein. Es sind Felswände.

Wandhänge und Schutthalden an deren Fuß bilden ein korrelates Paar. Die Wandhänge liefern den Schutt, der sich am Fuß anhäuft, entweder direkt oder dann aus Schuttrinnen, die so steil sind, daß der Schutt auch ohne Wasser auf die Halde gelangt. Aus der Art der Schüttung ergeben sich: 1. Schutthalden im engeren Sinn oder einfache Schutthalden am Fuß von geschlossenen Wänden. 2. Schuttkegel am Ausgang von Steinschlagrinnen. 3. Zusammengesetzte Schutthalden, wenn die Schuttkegel so eng benachbart sind, daß sie sich gegenseitig verschneiden und miteinander verschmelzen.

\section{Elemente zu einer Klassifikation von Schutthalden}

a) Als erstes Prinzip für eine Klassifikation kann der Zustand des Materials dienen, und zwar derjenige, in welchem es in die Schutthalde gerät. Durch das Material wird der maximale Böschungswinkel gegeben, ein Hauptcharakteristikum der Schutthalden.

b) Maßgebend für den Zustand des Materials ist die Form des Obergehänges und die Art der Schuttlieferung. Der Schutt kann durch direkten Sturz aus einer Wand oder aus einer Steinschlagrinne auf die Halde geraten. Das Material vermag zu 
stürzen und sich dabei wesentlich zu verändern oder bloß zu gleiten.

c) Für die Gestaltung der Halde sind weiter das Klima zu beachten, und da es sich um das Lokalklima handelt, die Exposition, die Abflußverhältnisse, die Schneebedeckung und der Feuchtigkeitsgehalt der Schutthalde. Mit dem Klima steht endlich die Vegetation in engstem Zusammenhang.

d) Von wesentlichem Einfluß ist die Art der Unterlage, auf die geschüttet wird.

e) Endlich hängen Form und Aussehen von Alter und Ablauf der Schüttungsvorgänge $a b$.

Diese fünf Faktorengruppen und die damit verknüpften Erscheinungsformen sollen nun der Reihe nach besprochen werden.

\section{Die einzelnen Faktorengruppen mit ihren Erscheinungsformen}

\section{Das Schüttungsmaterial und der maximale Böschungswinkel}

Jede Schutthalde hat ihr typisches Korngrößenspektrum. Es gibt Schutthalden mit auffallend gleichgroßen Bruchstücken und andere mit ausgeprägter Streuung nach Form und Größe. Wir unterscheiden Blockschutthalden von solchen mit mittlerem oder sehr feinem Korn. Besonders bei Schutt, der tonig verwittert, können Halden entstehen, die vorwiegend aus psamitischem und pelitischem Material aufgebaut sind und dann auch nicht das typische Verhalten von kohäsionslosem Material aufweisen, sondern rutschempfindliche Halden bilden.

Im allgemeinen fallen allerdings die Schutthalden durch den ausgeglichenen Böschungswinkel auf und unterscheiden sich von Schuttmantelhängen mit Kriech- und Gleitvorgängen, die durch ihre unruhige Oberfläche gekennzeichnet sind.

Der ausgeglichene Neigungswinkel ist ein Ausdruck der freien Schüttung von Einzelkomponenten oder kleinen Portionen groben Trümmermaterials, wodurch sich ein vom Material abhängiger maximaler Böschungswinkel herausbildet. Nach Piwowar (1903) ist dieser Winkel im obersten Achtel etwas größer und im untersten Achtel etwas kleiner als in den Dreivierteln des Mittelteils. Die Neigungsangaben beziehen sich auf den Mittelteil. Es gibt aber auch
Halden, bei denen der Scheitel etwas flacher und der Fuß steiler erscheinen.

$\mathrm{Da}$ die Oberflächenneigung mit verhältnismäßig einfachen Mitteln beobachtet werden kann, liegen viele Meßresultate vor. (PIWOWAR, 1903; STINY, 1925; HEIM, 1932; FREISE, 1933; JÄCKLI, 1957).

Je nach Form und Rauhigkeit der Schuttstücke schwankt der Böschungswinkel zwischen $26^{\circ}$ und $43^{\circ}$, streut also über $18^{\circ}$. Verwittert das Material sehr rasch, wie etwa bei Tonschiefern oder gar Schiefertonen, so können noch kleinere Werte auftreten. PIWOWAR, der über 250 Schutthalden der Alpen untersucht hat, gibt folgende Zusammenfassung:

I. Die Maximalböschung hängt von der Bruchart ab. Je massiger, eckiger, grobkörniger und rauhbrüchiger das Gestein, desto steiler die Halde. Je plattiger, schiefriger, rundlicher, je feinkörniger oder dichter, je milder die Bruchfläche, desto kleiner der Böschungswinkel. Schutthaldenböschungen sind ein direktes $M a ß$ für die Reibung der Trümmer. Man findet folgende Werte:

Körnig, rauhbrüchig, massig

Granite $37^{\circ}$

Sernifite

(Konglomerat) $35 \frac{1}{2}{ }^{\circ}$.

Kalksandstein $341^{\circ}$.

Körnig, rauhbrüchig, schiefrig

Gneise $34^{\circ}$

Glimmerschiefer $30^{\circ}$

Dicht, glattbrüchig, schiefrig

Tonschiefer $27 \frac{1}{2}^{\circ}$

Dicht oder fast dicht, glattbrüchig, massig

Etwas körnig (Schrattenkalk) stark $32^{\circ}$

Ganz dicht (Malm) schwach $32^{\circ}$

II. Gesteine von glatten Schieferungsflächen und plattigen Bruchstücken ergeben konstantere Böschungswinkel als solche von massigem Bruch ohne Schieferung:

$\begin{array}{lllc}\text { Schiefrige Gesteine } & \text { Max. } & \text { Min. } & \text { Diff. } \\ \text { Gneis } & 34 & 33 & 1 \\ \text { Glimmerschiefer } & 32 & 28 & 4 \\ \text { Tonschiefer } & 30 & 26 & 4 \\ \text { Massige Gesteine } & & & \\ \text { Granit } & 43 & 32 & 11 \\ \text { Hochgebirgskalk } & 351 / 2 & 28 & 71 / 2 \\ \text { Kreidekalk } & 36 & 28 & 8\end{array}$


III. Wenn rauhbrüchiger und glattbrüchiger Schutt gemischt sind, so ergibt sich ein Mittelwert:

Bündnerschiefer allein $27^{\circ} 41^{\prime}$

mit Kalk und Quarzit $29^{\circ} 40^{\prime}$

IV. Der Einfluß der Sturzhöhe ist gering. Bei benachbarten Kegeln unter verschieden hohen Wänden ergeben größere Sturzhöhen höchstens Unterschiede von $12^{\circ}-1^{\circ}$.

V. Im Beobachtungsmaterial lassen sich zwischen Nord- und Südexposition keine Unterschiede erkennen.

VI. Die Bewachsung beeinflußt die Haldenneigung nicht wesentlich. Bei Kalkstein ist die Böschung eher größer, wohl durch die Verfestigung durch Wurzeln und Humus. Bei Gneisen und Graniten sind bewachsene Halden weniger steil.

VII. Im Innern wasserreiche Kegel sind flacher, da das Wasser die Reibung herabsetzt. Bei kleintrümmerigem, schiefrigem und plattigem Material ist der Einfluß größer als bei grobblöckigen massigen Trümmern.

Bei Bündnerschiefern sind durchnäßte Kegel ca. $7^{\circ}$ flacher, bei Granit kaum $1^{\circ}$.

VIII. Die Trümmer ordnen sich nach der Größe. Am Scheitel sind die kleineren, am Fuß die größeren, wobei Blöcke oft bis über den Haldenfuß hinausspringen.

IX. Die durchschnittliche Größe der Trümmer hat keinen merklichen Einfluß auf die Böschung. Ein Kegel aus kleinem Gneisschutt hat $34^{\circ}$ Neigung, wie einer aus Kubikmeterblöcken.

Im Verlauf der Untersuchung werden wir auf einzelne Punkte dieser Zusammenstellung noch zurückkommen.

\section{Form des Obergehänges und Art der Schuttlieferung}

Bei der Form des Obergehänges und der Art der Schuttlieferung sind zunächst zwei Fälle zu unterscheiden, die dann wiederum differenziert werden können. Der Schutt gelangt im ersten Fall direkt aus dem Obergehänge in die Schutthalde oder er wird im zweiten Fall zunächst in einer Rinne gesammelt und erst aus dieser auf die Halde geschüttet.

Bei direkter Schuttlieferung ist eine geschlossene Wand die Voraussetzung, an deren Fuß sich eine durchlaufende Schutthalde bildet. Wenn die Wand sehr steil und hoch ist, so sind zwei Parteien zu unterscheiden (Fig. 1), nämlich die freie Wand, aus der der Schutt in die Halde stürzt und der Wandfuß, aus dem der Schutt in die Halde absitzt oder abgleitet. Je nach Zustand und Material der Wand prasseln aus dieser Kleinschutt (am extremsten aus Kieswänden) oder Grobschutt bis zu Riesenblöcken in die Halde. Die Lieferung kann permanent erfolgen oder sehr sporadisch sein. Brechen größere Partien los, kommt es zum Absturz ganzer Wandpartien oder zum Zusammenbruch von Vorsprüngen oder Türmen, sind alle Übergänge bis zu Bergstürzen gegeben. Diese Sturzart unterscheidet sich vor allem dadurch, daß nicht mehr Einzelkomponenten in die Halde geraten und hier zur Ablagerung kommen, sondern daß Schuttströme entstehen, die als geschlossene Masse abgleiten und stürzen, und sich auch weit über den Haldenfuß. ins Vorgelände ergießen. Bergstürze - als Gleitstürze - setzen auch keinen Wandhang voraus. Viele der großen Bergstürze haben erstaunlich geringes «Fahrgefälle» vom Abrißrand bis zum Schuttende, bei den Bergstürzen von Frank (Alberta, Kanada) und Elm $14^{\circ}$ und $16^{\circ}$, bei dem von Flims $8^{\circ}$. Das Aussehen der Ablagerung hat mit einer Schutthalde mit dem charakteristischen maximalen Böschungswinkel nichts mehr zu tun. In vielen Schutthalden gelangen einzelne große Blöcke, besonders wenn sie kubische Form haben und ins Rollen und Springen geraten, ins Vorfeld der Schutthalde. Auf die Mechanik der Schüttung wird in einer andern Arbeit eingegangen (GERBER und SCHEIDEGGER 1972).

Durch den Sturz wird beim Aufprall sowohl der Sturzblock wie das Sturzmaterial angeschlagen und teilweise zertrümmert. Es entstehen kleinere Komponenten und viel Feinmaterial in Form von Staub, der sich auf der Halde niederschlägt und bei der Konsolidierung eine wichtige Rolle spielt. Vom Wandfuß gelöster Schutt gleitet mit wenig Bewegungsenergie auf die Schutthalde und wird dabei in der Form kaum verändert. Größere Blöcke oder Platten von der Wandbasis sinken oft zunächst nur in das Lockermaterial des Wandscheitels ein und 
kommen dann erst ins Gleiten. Aus Kleinwänden wird oft nur Gleitschutt geliefert.

Es gibt auch Schutthalden, die ihr Material nicht aus einer Steilwand, sondern aus einem felsdurchsetzten Hang erhalten, aus dem die Schuttstücke vor allem abgleiten (Fig. 2) und damit die Form weitgehend beibehalten.

Bei der indirekten Schuttlieferung, bei der der Schutt aus einer Rinne in die Schutthalde gelangt, kommen beide Bewegungsarten, sowohl der Sturz, wie das Gleiten vor. Diese Rinnen können weite oder enge Schuttkanäle bilden, wobei manchmal wie bei den Wildbachanrissen, zu denen zudem Übergänge bestehen, Einzugstrichter, eine Durchflußrinne und ein Ablagerungsgebiet zu unterscheiden sind (Fig. 3). Bei engen Rinnen kann das Material vorübergehend durch einen größeren Block gestaut und dann, wenn das Hindernis nachgibt, in einem Schub in die Halde gelangen. Vom Aufbau einer Schutthalde, welche durch Absturz von Einzelstücken und kleineren Partien aufgebaut wird, unterscheiden sich solche Schuttkegel dadurch, daß deutliche Schuttströme erkennbar sind. Diese folgen am häufig trichterförmig erweiterten Ausgang der Schuttrinne mit Vorliebe dem Fels, so daß hier oft zwei Rinnen entstehen. Schon 1797 schrieb FABRE «Les roches attirent les eaux", das gilt auch für Schuttströme, wohl vor allem, weil dem Fels entlang der Schutt besonders gut abgeleitet und eine Rinnenbildung erleichtert (v. WISSMANN, 1951).

\section{Klima, Exposition, Durchfeuchtung und Vegetation}

Wir beschränken uns hier auf das humide Klima und die Höhenstufen der Alpen. Für die Schutthalden und ihr Aussehen ist, wie für alle Oberflächenformen und die sich darauf abspielenden Vorgänge, nicht das allgemeine Klima der freien Atmosphäre, damit also auch nicht die klimatische Schneegrenze, sondern das durch die orographischen Verhältnisse gegebene Klima der Bodenoberfläche und der bodennahen Luftschicht, das Mikroklima, verantwortlich. Für den Haldenaufbau im Gebirge spielt die Schneebedeckung eine besondere Rolle, da diese als Fläche dient, über die der Schutt abgleiten kann. Zudem erfolgt am Fuße von Wänden und besonders in Steinschlagrinnen eine Akkumulation von Schnee, der aus den Wänden abrieselt, so daß der Schutthaldenscheitel und der Ausgang von Rinnen oft bis weit in den Sommer hinein von Schneeresten bedeckt bleiben. Dies führt zu besonders starker Durchfeuchtung am Scheitel. Dadurch wird das Setzen und die Konsolidierung des Materials gefördert. Oft läßt sich am Haldenscheitel der Wand entlang eine kleine Verflachung von meist kaum einem Meter Breite beobachten, welche von Mensch und Tier als Fußweg benützt werden kann. Solche Verflachungen dürften aber kaum je durch menschliche oder tierische Beeinflussung zustande gekommen sein. Sie sind vielmehr als Materialsetzung der Felswand entlang zu deuten (Fig. 4). Dadurch wird wiederum der Schneeabsatz am Scheitel erleichtert. Es ist klar, daß der Schneeinfluß sich in nordexponierten Hängen wesentlich länger geltend macht, als in Südexposition und Nordexposition im allgemeinen feuchtere Halden ergibt.

Klima, Exposition und Durchfeuchtung bestimmen auch die Vegetation. Viele ältere Schutthalden der tieferen Höhenstufen sind bewaldet, während sie in der Nivalstufe kaum besiedelt oder sogar völlig kahl sind, was für die Umgestaltung durch Erosion von entscheidendem Einfluß ist. Denn, wenn kahle Halden oft Regenrillen zeigen, fehlen diese in den Vegetationsstufen oder treten doch zurück.

Durch das orographische Klima sind zwei für die Ökologie der Pflanzen wichtige Grenzen gegeben, die auch für die Gestaltung der Schutthalden von Bedeutung sind, die Waldgrenze und die Schneegrenze, wobei beide, wie das Schema von ELLENBERG (1963) zeigt, nicht als scharfe Linien, sondern als Übergangszonen in Erscheinung treten (Fig. 5).

Nach der Vegetation können wir also unterscheiden:

1. Kahle Schutthalden und-kegel

a) Klimatisch bedingt kahl. Nur durch Pionierpflanzen sporadisch besiedelt.

b) Durch intensive Schuttlieferung solange kahl, bis diese nachläßt und der Schutt besiedelt werden kann.

\section{Bewachsene Schutthalden und-kegel}

a) Mit Pionierpflanzen und alpinem Rasen und einzelnen Sträuchern und Krüppelbäumen.

b) Bewaldet.

Über die kahlen Schüttungen ist hier weiter nichts zu sagen, so daß wir uns der Schuttflora zuwenden können. 
Fig. 1: Die zwei Partien einer Felswand

a) freie Wand die Sturzschutt, b) Wandfuß der vorwiegend Gleitschutt liefert.

Fig. 2: Felsdurchsetzter Wandhang mit Absätzen. Liefert vorwiegend Gleitschutt.

Fig. 3: Schuttlieferung aus Steinschlagrinnen.

3a) f Fallschutt, g Gleitschutt.

3b) S Schuttsammeltrichter, R Abflußrinne, K Schuttkegel.

Fig. 4: Setzung des Schuttes an der Haldenstirn. Entlang der Felswand bildet sich ein Absatz, evtl. eine kleine Mulde.
Fig. 5: Schema des Formations-Mosaiks der Vegetation. Obere montane bis nivale Stufe der Alpen. Aus Ellenberg (1963).

Fig. 6: Wurzelschichtung in einem dichten Bestand der Kalkschuttflur. Thlaspeetum rotundifolii am Glärnisch 2250 m. Nach Jenny-Lips (1930).

1 Thlaspi rotundifolium, 2 Trisetum distichophyllum, 3 Viola cenisia, 4 Leontodon montanus, 5 Silene alpina. A Kohäsionsloser Oberflächenschutt, B Feinerdige Nährwurzelschicht, C Kohärenter, weitgehend verfestigter Schutt. Aus Ellenberg (1963).
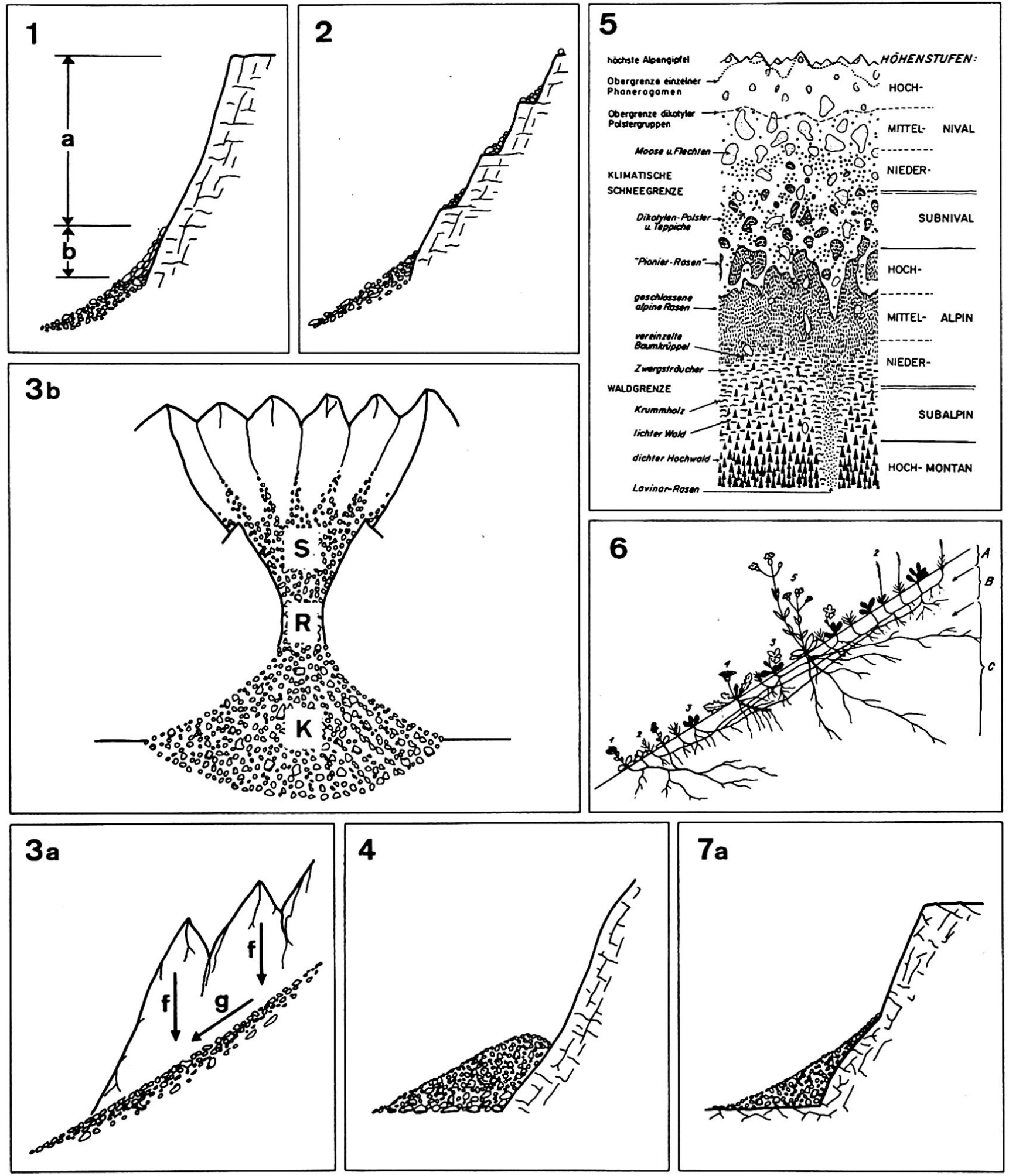
Fig. 7a: «Klassisches" Schutthaldenmodell. Konvexer Felskern unter der Schutthalde.

Fig. 7b: Schutthalde mit Gesteinswechsel.

Fig. 8: Schutthalden aus gegenüberstehenden Hängen.

Fig. 9: Umlenkung der Schuttströme durch Anpassung an die Neigung der Unterlage. a) Ansicht, b) Grundriß mit Höhenlinien.
Fig. 10: Überguß-Schutthalde. Der Fallschutt bildet nur eine dünne Decke und ruht dem verwitferten Material der Unterlage auf.

Fig. 11: Überguß-Schutthalde. Die Schuttströme erreichen den Haldenfuß nicht, so daß sie nicht gleich lang sind.

Fig. 12: Schutthalde auf Verflachung zwischen zwei Felswänden.

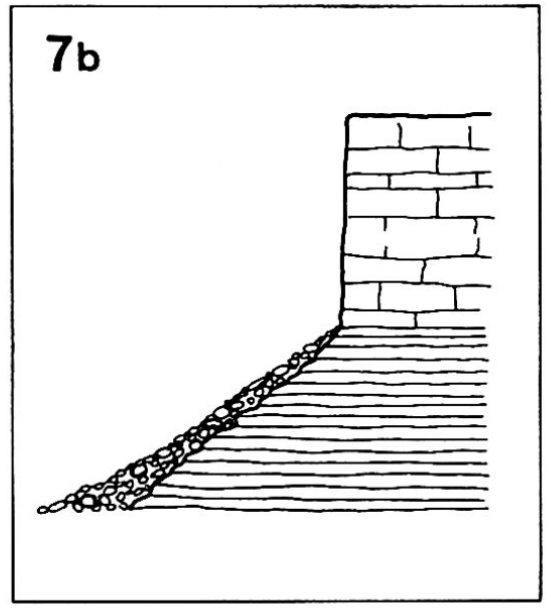

9 a

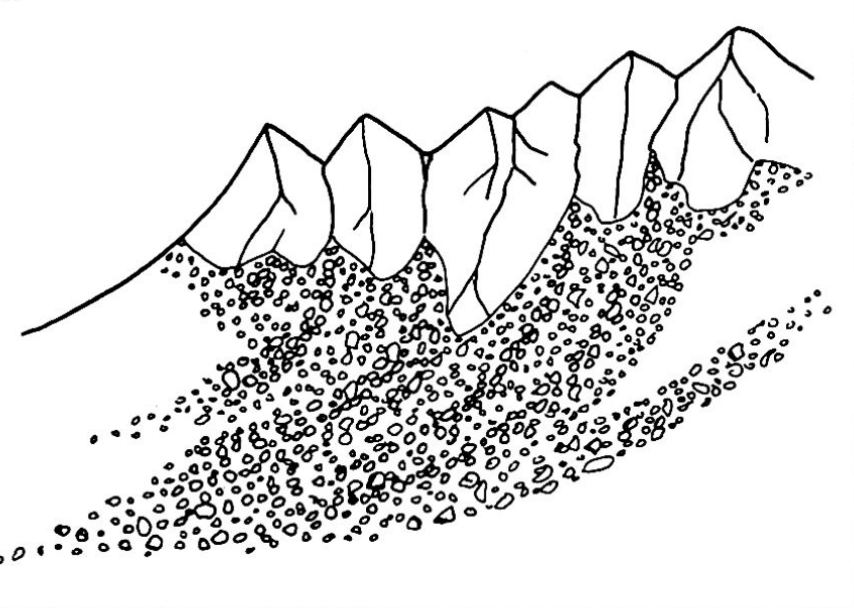

10

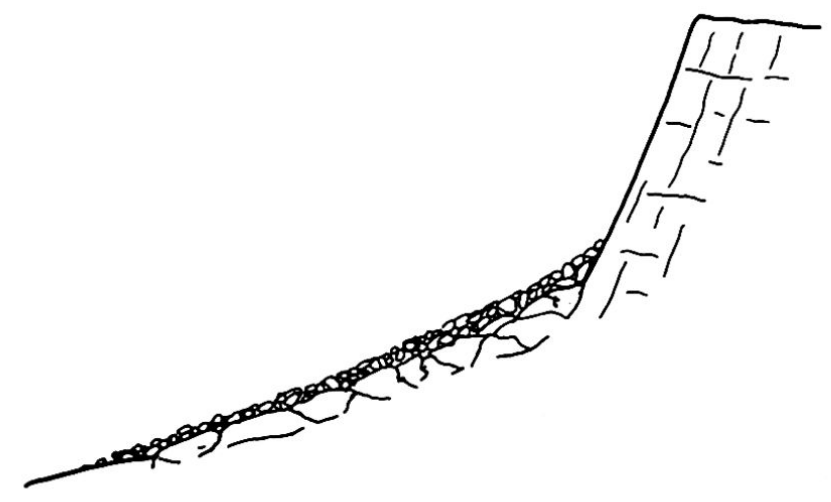

8

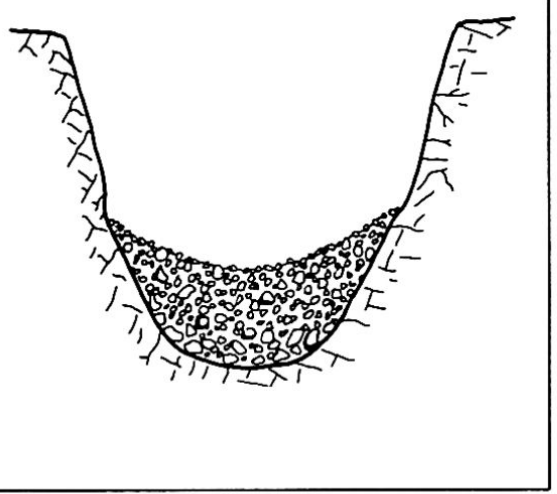

12

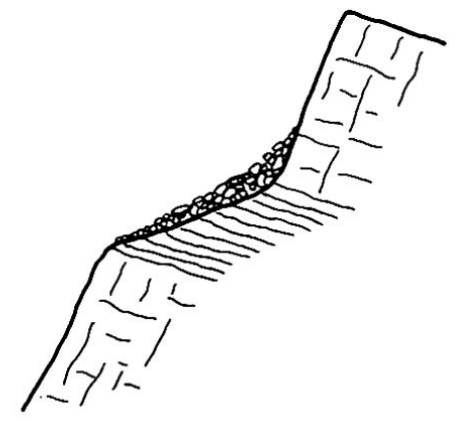

$9 b$

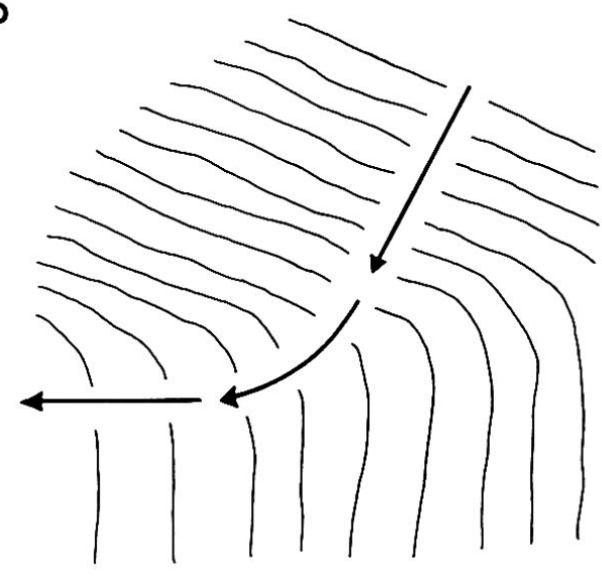

11

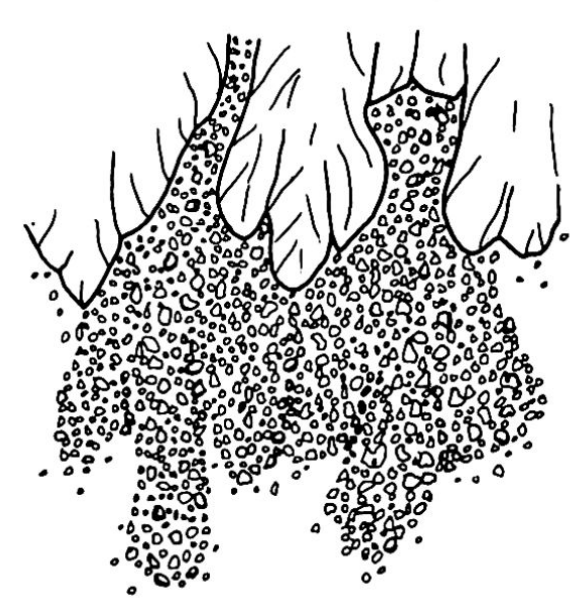


Auch bei sonnigen Halden gelangt man beim Graben unter einer trockenen und weitgehend sterilen Deckschicht zu feuchten Stellen, wo an den Trümmern nicht nur Staub haftet, sondern schon eine erste Besiedlung mit Bodenbakterien und Bodenalgen, Erdflechten und Erdmoosen einsetzt und damit eine Umwandlung des Gesteinsstaubes in den Humus enthaltenden Boden beginnt. Damit ist die erste Voraussetzung gegeben, daß sich Pionierpflanzen ansiedeln können.

Auf die vielfältigen und für das Aussehen einer Schutthalde wichtigen Vorgänge der Besiedlung kann hier nicht näher eingegangen werden und wir verweisen auf die Spezialliteratur (ELLENBERG 1963). SCHRÖTER (1932) unterscheidet 5 Gruppen von Schuttsiedlern (Fig. 6):

I. Gruppe: Die Schuttwanderer, mit verlängerten, horizontalen oder aufsteigenden Kriechtrieben, den Schutt durchspinnend, der Bewegung ausweichend.

II. Gruppe: Die Schuttkriecher, mit schlaffen oberirdischen Trieben, die von einem Punkt ausgehend auf dem Schutt liegen.

III. Gruppe: Die Schuttstrecker, die sich durch Strekken aufrechter Organe (Grundachsen, Stengel, Blattstiele) durch den Schutt arbeiten.

IV. Gruppe: Die Schuttdecker breiten niedere Rasendecken aus schlaffen wurzelnden Zweigen an der Schuttoberfläche aus und bilden so kleine, blühende Inselchen.

V. Gruppe: Die Schuttstauer pflanzen sich mit festen Horsten in den Schutt und stauen ihn.

Durch fortschreitende Sukzessionen bewalden sich die Schutthalden. In hohen Lagen, oberhalb der Waldgrenze, kommen Zwergsträucher und niederliegende Bäume hoch, so die krautige Weide (Salix herbacea L., "arbor minima» nach Linné) Alpenrosen, Alpenerlen und Legföhren. In tiefen Lagen entwickelt sich Hochwald.

Bevor Schutthalden oder -Kegel völlig bewachsen sind, wächst die Vegetation zwischen aktiven Schuttströmen zungenförmig in die Höhe. Wie PIWOWAR (1903) zusammenfaßt, hat die Bewachsung keinen großen Einfluß auf den Böschungswinkel. Immerhin stellt der Autor fest, daß in Kalkschutthalden eher etwas größere Böschungswinkel auftreten, was auf petrographische Beeinflussung hinweisen könnte. In Waldungen staut sich hinter jedem Baum Schutt auf, gefallene Bäume wirken wie Barrieren und jeder Stamm zeigt bergwärts Schlagspuren. Durch die Bäume wird der Schuttfluß stark behindert.

\section{Art der Unterlage}

Bei der Beurteilung des Einflusses der Unterlage, auf die geschüttet wird, sind drei Gesichtspunkte zu berücksichtigen.

1. Die Form der Unterlage.

2. Die Höhenausdehnung der Unterlage.

3. Das Material der Unterlage.

Die einfachste Form der Unterlage besteht dort, wo die Haldenbasis einer Horizontalebene aufliegt (Fig. 7a). Diese kann irgend eine horizontale Hangfläche, also eine Terrasse, in vielen Fällen aber auch der Talboden sein. Das Rückwittern einer Plateaufelswand und die Form des Felskerns unter der Schutthalde wurde schon mehrmals theoretisch untersucht (FISCHER 1866, LEHMANN 1933, BAKKER 1947). Eine kritische Würdigung dieser Theorien wurde von GERBER und SCHEIDEGGER (1973) publiziert. Wie schon in dieser Arbeit betont wurde, ist die Annahme einer Plateaufelswand, die einer Horizontalebene aufruht, eher selten verwirklicht. Auch kann sich unter der Felswand ein Sockel eines anderen Gesteins verbergen (Fig. 7b). Im Idealfall einer homogenen Felswand und ungestörter Ablagerung des Fallschuttes entsteht unter dem Schutt und von diesem geschützt ein parabelähnlich vorspringender Felskern. Oft wird aber, wenn in einen Talboden geschüttet wird, vom Haldenfuß Material entfernt. Ist der Schutt schon konsolidiert, so wird die Halde unterschnitten, wenn nicht, so kommt die kohäsionslose Schuttdecke ins Nachrutschen. Beim Zurückwittern der Wand entsteht dann im Idealfall eine Felsfläche, die nur lose von Schutt bedeckt ist, die LEHMANN (1933) als "Richtersche Denudationsböschung» bezeichnet hat.

Erreicht die Schutthalde den Gegenhang, so wird das Talgewässer an diesen gedrängt, gestaut und in die Höhe gehoben, wenn die Tiefenerosion nicht Schritt halten kann. Besonders wirkungsvoll ist die Aufschüttung, wenn ein Talgewässer fehlt und aus beiden Hängen geschüttet wird (Fig. 8). 
Ist der Talboden stark geneigt oder erfolgt die Schüttung seitlich einen Hang hinein, so wird das Schuttmaterial in die Tal- oder Hangrichtung umgelenkt (Fig. 9). Der Fall- und Gleitschutt beschreibt dann, seiner Bewegungsenergie entsprechend, gekrümmte Bahnen. Sehr häufig ruht die Wand einem potentiellen Schuttmantelhang auf, dessen Höhe so ausgedehnt ist, daß der Fallschutt den Fuß nicht erreicht.

Dieser Hang kann zudem aus einem anderen Material bestehen als die schuttliefernde Wand. Es kann ein Felsoberbau und ein Schuttmantelhang als Unterbau oder Sockel unterschieden werden (Fig. $10,11)$, Der Schutt der Wand bildet auf der Oberfläche des Unterbaues eine Grobschuttdecke aus Fremdmaterial. Auf relativ steilen Unterbauhalden ist diese Schuttdecke nur wenig mächtig, so daß von einer Übergußschutthalde gesprochen werden kann. Ist die Halde in Bezug auf die Felswand hoch, so reicht die Schuttdecke nicht bis zum Haldenfuß. Dann ist die Untergrenze der Schutthalde oft nicht scharf begrenzt. Besonders, wenn der Schutt aus Steinschlagrinnen stammt, entwickeln sich einzelne Schuttströme, die besonders weit hinabreichen.

Besteht die Unterlage, auf welche der Schutt abgelagert wird, aus rutschempfindlichem Material, so kommt durch die zusätzliche Belastung die Unterlage und damit auch die Schutthalde ins Rutschen und es entstehen Oberflächenformen, die einer Halde aus kohäsionslosem oder doch kohäsionsarmem Material völlig fremd sind. Die sonst für Schutthalden typische einheitliche Oberfläche wird durch

Faktoren zur Klassifikation von Schutthalden

\begin{tabular}{|c|c|c|c|c|c|c|}
\hline Stückgröße & $\begin{array}{l}\text { Form und } \\
\text { Oberfläche } \\
\text { der } \\
\text { Schuttstücke }\end{array}$ & $\begin{array}{l}\text { Ober- } \\
\text { gehänge }\end{array}$ & $\begin{array}{l}\text { Klima, } \\
\text { Exposition, } \\
\text { Feuchtigkeit }\end{array}$ & Vegetation & Unterlage & Stadium \\
\hline $\begin{array}{l}\text { Blockhalde } \\
\text { mittlere } \\
\text { Psamite und } \\
\text { Psephite } \\
\text { viel } \\
\text { pelitisches } \\
\text { Material }\end{array}$ & $\begin{array}{l}\text { körnig, } \\
\text { rauhbrüchig, } \\
\text { massig } \\
\text { körnig, } \\
\text { rauhbrüchig, } \\
\text { schiefrig } \\
\text { dicht, glatt- } \\
\text { brüchig, } \\
\text { massig } \\
\text { dicht, glatt- } \\
\text { brüchig, } \\
\text { schiefrig }\end{array}$ & $\begin{array}{l}\text { geschlos- } \\
\text { sene, steile } \\
\text { hohe Wand: } \\
\text { Sturzschutt } \\
\text { niedrige } \\
\text { Wand: Gleit- } \\
\text { schutt } \\
\text { flache Wand, } \\
\text { felsdurch- } \\
\text { setzter Hang: } \\
\text { Gleitschutt } \\
\text { Wand mit } \\
\text { Kluftgassen } \\
\text { und Stein- } \\
\text { schlagrinnen: } \\
\text { Oft strömende } \\
\text { Schüttung }\end{array}$ & $\begin{array}{l}\text { viel Nieder- } \\
\text { schlag } \\
\text { trocken } \\
\text { viel Schnee } \\
\text { wenig } \\
\text { Schnee } \\
\text { Nord- } \\
\text { exposition } \\
\text { Süd- } \\
\text { exposition }\end{array}$ & $\begin{array}{l}\text { kahle } \\
\text { Schutthalde: } \\
\text { Klimabedingt } \\
\text { kahle } \\
\text { Schutthalde: } \\
\text { durch } \\
\text { intensive } \\
\text { Schüttung } \\
\text { bedingt } \\
\text { bewachsene } \\
\text { Schutthalde: } \\
\text { Pionier- } \\
\text { pflanzen } \\
\text { Rasen } \\
\text { Wald }\end{array}$ & $\begin{array}{l}\text { Schüttung } \\
\text { bis in Flach- } \\
\text { boden, Wand } \\
\text { ursprünglich } \\
\text { bis Flach- } \\
\text { boden } \\
\text { Schüttung } \\
\text { auf Hang, } \\
\text { Stirne bis } \\
\text { Hangfuß, } \\
\text { Stirne endet } \\
\text { im Hang } \\
\text { Überguß- } \\
\text { schüttung } \\
\text { Schüttung } \\
\text { auf Absatz } \\
\text { zwischen } \\
\text { Felswänden }\end{array}$ & $\begin{array}{l}\text { Embryonale } \\
\text { Schutthalde } \\
\text { Schutthalde } \\
\text { in raschem } \\
\text { Aufbau } \\
\text { nachlassende } \\
\text { Schuttlieferung } \\
\text { keine Schutt- } \\
\text { lieferung mehr }\end{array}$ \\
\hline
\end{tabular}


Teilrutsche gegliedert. Es entstehen Absätze und Wülste, kurz, all die für Rutschhänge charakteristischen Formen.

Wird die Schutthalde auf einer Verflachung zwischen zwei Wandhängen aufgebaut, so kann sie nur so lange anwachsen, bis der Schuttfluß die untere Wand erreicht. Weiterer Schutt stürzt dann über diese ab (Fig. 12). Im allgemeinen wird sich beim Rückwittern der beiden Wände eine Richtersche Denudationsböschung im Anstehenden entwickeln, die nur von einer dünnen Schuttstreu bedeckt ist.

\section{Alter und chronologischer Ablauf der Vorgänge}

Jede Schutthalde entwickelt sich aus embryonalen Anfängen bis zu einem Endzustand, bei dem die Schuttzufuhr nachläßt und dann, wenn die Wand über der Schutthalde völlig zerfallen ist oder vom höhersteigenden Schutt bedeckt wird, ganz aufhört. In den Alpen hat der Aufbau vieler Schutthalden mit dem Rückzug der Gletscher begonnen. Wenige sind jünger.

Nach dem Alter können also junge, mitten im lebhaften Aufbau sich befindliche und alte, nicht mehr wachsende Schutthalden unterschieden werden.

Die Entwicklung ist nicht kontinuierlich. Auf stürmisches Wachstum können Ruhepausen, durch den Zusammenbruch eines Wandteils wieder erneut rascher Aufbau folgen. Die Schuttlieferung ist auch nach der Form und Größe des Materials verschieden. Bei größeren Abbrüchen liefert die Wand Blockmaterial, dann oft wieder lange nur Material von Grobkiesgröße. Dementsprechend wechselt auch das Aussehen der Halde.

\section{Verdankungen}

Die Arbeit ist eines der Ergebnisse der Untersuchungen, welche vom Institut für Geophysik der Technischen Hochschule Wien unter der Leitung von Herrn Prof. A. E. SCHEIDEGGER im Rahmen des internationalen Geodynamik-Projektes durchgeführt werden. Sie wurde von der Österreichischen Akademie der Wissenschaften durch das GeodynamikKomitee unter dem Vorsitz von Herrn Prof. F. STEINHAUSER unterstützt.

\section{Literatur}

ELLENBERG, HEINZ 1963: Vegetation Mitteleuropas mit den Alpen. Stuttgart (Eugen Ulmer)

FREISE, FRED W. 1932/33: Beobachtungen über Erosion an Urwaldsgebirgsflüssen des brasilianischen Staates Rio de Janeiro. Zschr. f. Geomorphologie, Bd. VII

GERBER, ED. und SCHEIDEGgER, A.E., 1972: Zur Felskernbildung unter Schutthalden. Petermanns Mitt.

GERBER, ED. und SCHEIDEGGER, A. E., 1972: On the Dynamics of Scree Slopes

HEIM, ALBERT, 1932: Bergsturz und Menschenleben. Viertelj. Schr. Natf. Ges. Zürich

JÄCKLI, HEINRICH, 1957: Gegenwartsgeologie des bündnerischen Rheingebietes. Beitr. z. Geologie der Schweiz Geot. Serie, Lief. 36

JENNY, HEINRICH, 1930: Vegetationsbedingungen und Pflanzengesellschaften auf Felsschutt. Beih. Bot. Zentralbl. Bd. XLVI, Dresden

LEhMANN, OTto, 1933: Morphologische Theorie der Verwitterung von Steinschlagwänden. Viertelj. Schr. Natf. Ges. Zürich LXXV

MORAWETZ, S. O., 1933: Beobachtungen an Schutthalden, Schuttkegeln und Schuttflecken. Geom. Zeitschr. VII

PIWOWAR, A., 1903: Über Maximalböschungen trokkener Schuttkegel und Schutthalden. Diss. Zürich

SCHEIDEGGER, A.E., 1970: Theoretical Geomorphology. Springer. Berlin

SCHRÖTER, CARL, 1932: Kleiner Führer durch die Pflanzenwelt der Alpen. Zürich

STINY, J., 1925/26: Neigungswinkel von Schutthalden. Zeitschr. f. Geom., Bd. I

WISSMANN v., H., 1951: Über seitliche Erosion. Colloquium geogr. 1, Bonn 